\title{
RoboGenius Team Description Paper Review and Develop AI Algorithms and Decision Making in Rescue Simulation Environment
}

\author{
Abbas Khosravi, Mohammad Kazem Farhadipour, Seyed Mojtaba Hosseinifar, and Najme Roozmand
}

\begin{abstract}
In this article we are trying to present some algorithms and strategies in order to improve the rescue simulation and decrease the number of damages and pains that happens in the rescue simulation environment. Since the activities of an agent can have a deserving effect on efficiency, so it should be done by various things, such as information that any agent can obtain from surrounding environment, information that can be obtained from communication with homogeneous agents and order them. If there is no such information, we are trying to use machine learning and cooperation without communication strategies. In our study, cooperation and path planning have main role.
\end{abstract}

Index Terms-Rescue simulation, algorithm, RoboCup, artificial intelligence.

\section{INTRODUCTION}

This article describes Genius rescue agent simulation team. Please Simulation system (RCRSS) is a distributed system that made from several connected units by a network. Geographical information system (GIS) provides initial circumstances of damaged environment. Viewer indicates the circumstances of damaged environment. Kernel unit controls the simulation and communications between units An RCR agent controls the action of an object in damaged environment and there are seven kinds of them: civilians, ambulance team, fire brigade, police force, ambulance center, fire station and police office. The ambulance team, fire brigade and police force are collectively called a platoon agent and ambulance center, fire brigade and police office called a center agent. These two agents are called a rescue agent. Rescue simulation environment is a multi-agent environment that every agent has its own responsibility. We explain the responsibility and the improvement of the efficiency of any agent in grace. We want to develop cooperation without communication idea and study an explorer agent, so choose Rescue Agent Simulation as test bed [1]. Before that we study and test cooperation without communication idea in 2D Soccer Simulation [2].

\section{DECISION MAKING FOR DIFFERENT AGENTS}

Given to the decision making is one of the important things

Manuscript received February 4, 2013; revised May 28, 2013.

Abbas Khosravi and Mohammad Kazem Farhadipour are with the Computer Engineering Department, University of Payam Noor, Shiraz, Iran (e-mail: Khosravi1356@Yahoo.com, Kazem_Farhadipour@Yahoo.com).

Seyed Mojtaba Hosseinifar is with the Industry Engineering Department, University of Payam Noor, Shiraz, Iran (e-mail: Hoseinifar.m@Gmail.com).

Najme Roozmand is with the Psychology Department, University of Payam Noor, Shiraz, Iran (e-mail: Roozmandn@Yahoo.com). that an agent can do, we use different priorities for different agents.

\section{A. Fire Brigade}

When several areas are on fire, choosing an important fire site is important. We should decrease the damages and injuries with strategies and the less number of fire brigade. We order the fire site according to these factors:

1) The number of neighbor buildings and area of them

2) The area of the burned building and the number of its floors

3) Position of the building in the map

4) How long it has been burning

5) The number of victims in the burning area

6) The number of agents that needs to extinguish the fire site

7) Type and temperature of the burned building

8) The priority in distinguishing fire is with ambulance team

9) If there is not enough time to extinguish the fire in one cycle, we should use the rest of the water to water down the neighboring areas of the fire. In order to decrease the temperature of the building and prevent spreading the fire

10) If there is not any fire that the fire brigades want to extinguish, we should divide the map into two sections and water down the distance between them.

\section{B. Ambulance Team}

In order to rescue victims soon, ambulance team considers these points:

1) How deep a civilian or an agent is buried

2) Percentage of their health (HP)

3) Percentage of damages and injuries

4) Time estimation that needs to reach the buried civilian and rescue it before dead

5) The average distance of buried civilian to the refuge and average distance of it to ambulance

6) If a fire agent buried or damaged it should be rescue first

\section{Police Force}

Police agents have a very important role in communication with other agents. Among these, we have very important roads that should be open first:

1) Roads between refuge and fire zone

2) Roads that connected different fire zones to each other

3) Main roads that connect different roads to each other

4) When there is only one road between two zones

5) Roads that have much traffic. It is clear that choosing a suitable block path to be opened by the police force, can have an important role in improving the team conditions as well as the final result. We developed our police agent in way that takes different decision in two different below 
situation:

6) When the ambulance and fire brigade agents have not explored in environment well yet.

7) When ambulance and fire brigade agents have taken special paths more. (Like Ant Colony problem)

In first state to solve that problem, we decided to represent the city as a graph, and use a Minimum Spanning Tree algorithm to find the best roads to be cleared. The goal of such this algorithm (like Prim and Kruskal algorithms) is to find the Minimum Tree that reaches all nodes in a graph, considering the weights of each edge [3]. The algorithm will be run for each agent, and the results sent to the police center, which will then inform the other agents, telling them which roads should be cleared. We used Prim's algorithm because it has better time complexity in graphs that have more edge. Its time complexity is $n^{2}$ where $n$ is number of nodes. Fig. 1 shows finding a sample Minimum Tree.

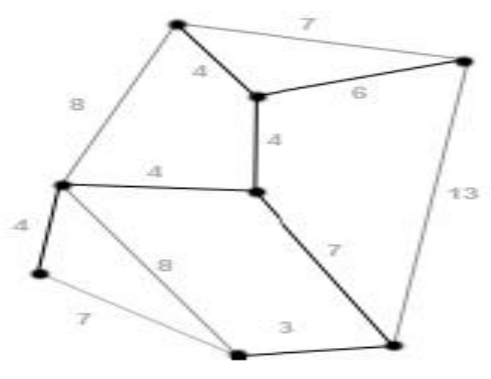

Fig. 1 Finding a sample minimum tree.

In second state, our police agent strategy is similar to first state strategy but to find the Minimum Tree, besides considering the weights of each edge, it considers the paths that ambulance and fire brigade agents have taken those more. Police agent uses formula 1 to update the weights of each edge, where $W_{\text {new }}$ is new weight, $W$ is current weight and $T_{\text {ratio }}$ is the ratio of ambulance and fire brigade agents transmission number and all ambulance and fire brigade agents transmission between two nods. After that it uses Prim's algorithm to find the Minimum Tree. This updating is caused that police agent finds a Minimum Tree with higher probability of ambulance and fire brigade agent's transmission. It reaches the goal that mention in beginning of this part.

$$
W_{\text {new }}=W-T_{\text {ratio }}
$$

\section{COMMUNICATION}

Given to the ability of the agents for understanding their environment is low, they share their received information to the extent possible. The communications between agents is one of the important points that can help our decision structure [4]. The agent obtains its needed information in two ways:

1) According to surrounding area

2) According to received messages from homogeneous agents Centers can communicate with each other and also agents with homogeneous centers and also with each agent.

\section{A. Message}

Sending a message is one of the important ways of obtaining information for world modeling. What is important in managing the messages is, given the limitation of the agents and centers in the number of messages that they can send and receive; they are able to order the messages according to their importance. We defined these priorities according to sender of the message and other points, agents and centers send and receive more important ones and also they save less important ones in their database unless they have been expired and they send them in next several cycles. Given to the length limitation of messages (256 Bytes), we use compress and decompress ways and relevant protocols.

\section{B. Cooperation}

The goal of multi agent systems in most cases is solving the complex problems by distribution it through different dimensions. In such systems a team of voluntary agents are able to achieve specified goals by collaboration. In most of such systems the agents are designed as existence with limited information and abilities so they can act rapidly and simply replace by others. The communication between two agents is necessary due to following two reasons:

Firstly facilitate the dispatching information to the agents which the necessary information is not available for them. Secondly the initiation of a collaboration strategy by diversified logarithm and procedures. It is evident that lack of communication or unsecured communications in a team of agents with common goal can rebuff achieving final goals. Some of reasons which cause the real or resembling multi-agent systems to repeat the unsecured communications or cut of communications can be numerated as following. Disorder and cut of communicational systems, Decrease in quality of communication due to miscommunication, High traffic of communication channel, Decrease of security of collaboration and risk causing, Lack of communicational infrastructure: For example cut of telecommunication can be happened in relief and rescue operations while a team of several polices and fire fighters are in a mission. In multi-agent systems cooperation between agents has a very important role, we want to do this cooperation in two ways, one of them is operating easily, cooperation through communication and the other is cooperation without communication. We express one example here. Imagine that the part of the city is on fire, since there is a possibility of blocked road in these areas and distance between refuge and roads near the fire, in the cooperation with communication polices are informed to go to these zones but in cooperation without communication when the police agents observe the fire and development of the fire or when they observe that several of agents are together in one place, without any previous request they automatically go to these areas and if the near roads are blocked, they will try to open them.

\section{PATH PlANNING}

One of the important points in rescue field is finding the best and the least expensive path for an agent to reach its target. Rescue agent simulator is a sequential, dynamic, nondeterministic and partially observable environment. This 
environment is the most difficult one for an explorer agent. This is a dynamic environment, since when an agent deciding to choose a target and doing its calculation, the environment changes. Facing such an environment, it's more suitable to use special algorithms such as A*, IDA* [5]. In order to have a desirable path planning we considered: A*, IDA*, DFS, BFS algorithms. For choosing suitable algorithm we look into consideration five following scales: standard examination result, optimality, complete, time complexity and space complexity. Our standard examination has main role in algorithm choice [3]. BFS and DFS: Although implementation of BFS algorithm is simple, its space complexity isn't optimizing. DFS algorithm doesn't lead to a suitable conclusion, because it isn't complete, although its space complexity is lower. These two algorithms, obtained 141.58 and 119.82 in an examination we carried out according our own standards. This examination calculates a result score (RS) by server average score (SAS), finding speed score (FS); blocking score (BS) features in formula 2. Result score (RS) has main role in our algorithm choice.

$$
R S=\frac{((2 \times S A S)+F S)}{B S}
$$

$\mathrm{A}^{*}$ : This algorithm is both complete and optimized, if it has admissible heuristic. Therefore it is reasonable to use it, because it obtained higher score than two other algorithms in our examination (453.3 scores). The weak point of $A^{*}$ was that when the distance between source and destination was increase, this algorithm faced memory problems and wasn't able to recognize the path. IDA*: According to what mentioned about $\mathrm{A}^{*}$, using this algorithm is more reasonable, because it solves the memory problem of $A^{*}$. Using this algorithm, we obtained suitable score in our examination (598 scores). According to what mentioned above and information in Fig. 2, we choose IDA* algorithm. Using information about the environment, we create a graph in our world model. Naturally by passing of time and knowing the agent and completing the information, this graph will be more complete and it will change. One of the limitation we faced in using IDA*, was the calculation of an optimized and effective heuristic function. We did different experimentation heuristic function to calculate optimized function. Be-cause our environment is graph so in some cases obtained function didn't give us the optimize path, we had to study monotone feature in addition to being admissible. According to the following formula 3 :

$$
f(n)=g(n)+h(n)
$$

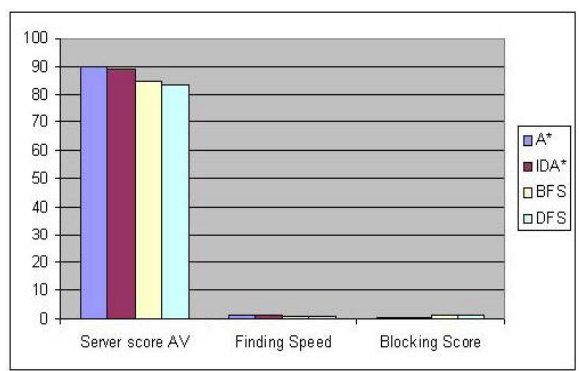

Fig. 2. Compare examination feature for different algorithms.

In which $f(n)$ is estimated cost of the cheapest solution and $g(n)$ is the cost of the path from the source node to $\mathrm{n}$ nod, and $h(n)$ is the estimated cost of the cheapest solution from $n$ node to destination node. Monotone feature means: it means that we always choose the shortest path to reach the target According to what we mention above and environment feature we use HSDl heuristic, which considers monotone feature because the straight path is the shortest distance between two points (Fig. 3 and formula 4). In formula $4 c$ ( $n$, $m$ ) is the cost of path between $n$ node and m node. This theoretical result showed hopeful result according the experimentation done.

$$
h(n)<=c(n, m)+h(m) .
$$

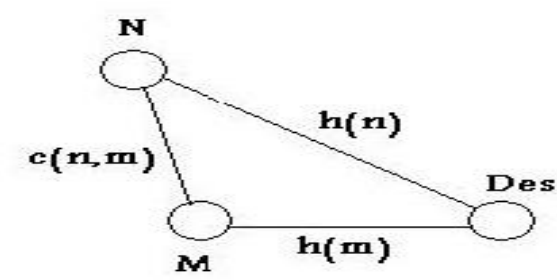

Fig. 3. Compare examination feature for different algorithms.

\section{SOLVING TRAFFIC PROBLEM}

Our rescue agents were usually blocked in a traffic jam. This problem rose since the rescue agents couldn't identify a traffic situation; therefore they stopped in their positions. To solve this problem we used a Decision Tree [6]. Such tree is used to identify a situation [7]. We considered the following features for this tree:

1) The amount of time of stability in a position

2) The distance to the nearest agent

3) The type of the road (busy road or normal road)

4) The amount time of stability of near agents Road blocking (i.e. is road blocked by blocking sub-simulator)

5) Agent type (ambulance, fire brigade, police)

6) Congestion of agent in special radius (for example 8 meter)

7) The amount of stability of near agents we used offline training for this tree by 750 training data and with the help of ID3 algorithm. We ran this tree on 100 maps and about $90.3 \%$ cases the agent was successful in identifying the traffic situation.

\section{RESCUEANALYZER}

Although it is believed that autonomous rescue agent is main idea in our team development, experience shows that would be helpful if team developers can instantly be aware of the general outcome of simulation operation with help of chart. According to what mention above we have designed, a simple application to able to provide the team developer with a chart after a simulation process. This application has been designed via Java language; it was used as a primary version in our laboratory. This application makes available the feature which is effective in calculating scores for the team in form of chart. This feature is as following: Civilian Rescue, amount of building area undestroyed, ratio of fires extinguished and et 
cetera. Fig. 4 shows a primary version of Rescue Analyzer.

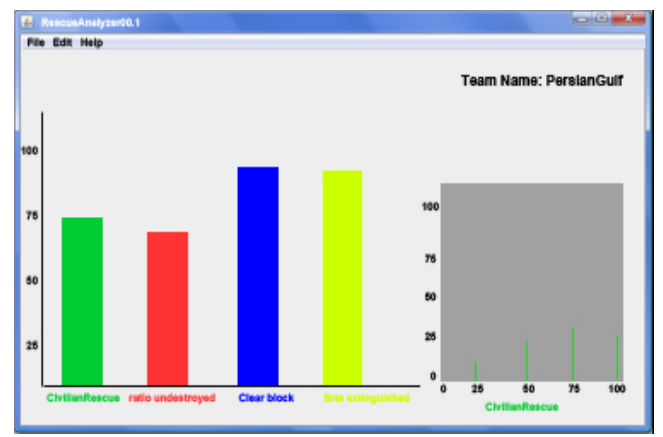

Fig. 4. Primary version of rescue analyzer.

\section{REFERENCES}

[1] M. R. Genesereth, M. L. Ginsberg, and J. S. Rosenschein, "Cooperation without communication," in Proc. Association for the Advancement of Artificial Intelligence, Stanford University, Palo Alto, California, 1986, pp. 51-57.

[2] R. de Boer, J. Kok, and F. Groen, "The uva trilearn 2001 robotic soccer simulation team," M.S. thesis, Dept. Artificial Intelligence and Computer Science., Amsterdam Univ., Amsterdam, Netherlands, 2001.

[3] T. H. Cormen, C. E. Leiserson, R. L. Rivest, and C. Stein, Introduction to Algorithms, 2nd ed. MIT Press \& McGraw-Hill, USA: New York, 2003, ch. 4, pp. 450-560.

[4] P. Stone and M. Veloso, "Communication in domains with unreliable, single-channel, low-bandwidth communication," in Proc. Collective Robotics, Berlin: Springer-Verlag, 1998, pp. 85-97.

[5] S. J. Russell and P. Norvig, Artificial Intelligence: A Modern Approach, Prentice-Hall, Upper Saddle River, New Jersey: Springer-Verlag, 1995, ch. 2.

[6] P. Stone and M. Veloso, "Using Decision Tree Confidence Factors for Multi-Agent Control," in AGENTS '98 Proc. the Second International Conf. on Autonomous Agents, New York, 1998, pp. 86-91.

[7] M. Main, Data Structures and Other Objects Using Java, Addison-Wesley, 1999, ch. 4.

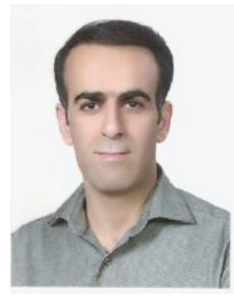

Abbas Khosravi was born in Iran in 1978. He has spent the bachelor's degree in Software Engineering, Shiraz University in 2000 and passes Master level IT engineering (software design and production trends) in Shiraz University. All of his studies had been in Iran. $\mathrm{He}$ is working at the university. He has presented numerous papers in the field of robotics. He likes to study Ph.D. outside of Iran. Khosravi has submitted numerous papers for various conferences where you can search for it

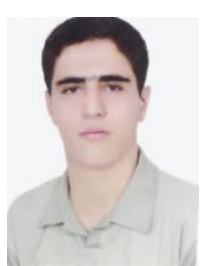

Mohammad Kazem Farhadipour was born in Iran in 1989. He has spent the bachelor's degree in Software Engineering, Payam Noor University of Shiraz in 2010 and passes Master level computer engineering (artificial intelligence) in Shiraz University. All of his studies had been in Iran. He is working at the university. He has presented numerous papers in the field of robotics. Farhadipour has submitted numerous papers for various conferences where you can search for it.

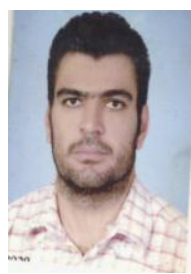

Seyed Mojtaba Hosseinifar was born in Iran in 1984 $\mathrm{He}$ has spent the bachelor's degree in industrial engineering in Azad University of Shiraz in 2008 and passes in Master level management in Payam Noor University of Tehran. All of his studies are in Iran. He is working at the university. He likes to study Ph.D. outside of Iran. Hosseini has sent far too many papers for various conferences that you can search for it.

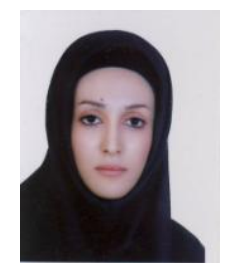

Najme Roozmand was born in Iran in 1988. She has spent the bachelor's degree in psychology Degree in Payam Noor University of Shiraz in 2012. All of the studies in Iran. She is not working. She is interested to study $\mathrm{Ph}$.D. Roozmand has sent far too many papers for various conferences that you can search for it. 